Acta Crystallographica Section E

Structure Reports

Online

ISSN 1600-5368

Andrea Johnston, a Alastair J. Florence $^{a *}$ and Alan R. Kennedy

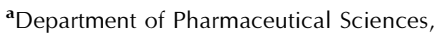
University of Strathclyde, 27 Taylor Street, Glasgow G4 ONR, Scotland, and ${ }^{\mathbf{b}}$ WestCHEM, Department of Pure \& Applied Chemistry, University of Strathclyde, 295 Cathedral Street, Glasgow G1 1XL, Scotland

Correspondence e-mail:

alastair.florence@strath.ac.uk

\section{Key indicators}

Single-crystal X-ray study

$T=123 \mathrm{~K}$

Mean $\sigma(\mathrm{C}-\mathrm{C})=0.003 \AA$

$R$ factor $=0.040$

$w R$ factor $=0.110$

Data-to-parameter ratio $=15.7$

For details of how these key indicators were automatically derived from the article, see http://journals.iucr.org/e.

\title{
Hydrochlorothiazide dimethyl sulfoxide solvate
}

Hydrochlorothiazide forms a 1:1 solvate with dimethyl sulfoxide, $\mathrm{C}_{7} \mathrm{H}_{8} \mathrm{ClN}_{3} \mathrm{O}_{4} \mathrm{~S}_{2} \cdot \mathrm{C}_{2} \mathrm{H}_{6} \mathrm{OS}$. The crystal structure contains a hydrogen-bonding network comprising three $\mathrm{N}-$ $\mathrm{H}$. O O contacts.

\section{Comment}

Hydrochlorothiazide (HCT) is a thiazide diuretic which is known to crystallize in at least two non-solvated forms; form I (Dupont \& Dideberg, 1972) and form II (Florence et al., 2005). The dimethyl sulfoxide (DMSO) solvate, (I), was produced during an automated parallel crystallization polymorph search on HCT. The sample was identified as a new form using multisample X-ray powder diffraction analysis of all recrystallized samples (Florence et al., 2003). Subsequent manual recrystallization by slow evaporation of a saturated DMSO solution at $278 \mathrm{~K}$ yielded samples of (I) suitable for single-crystal X-ray analysis (Fig. 1).<smiles>NS(=O)(=O)c1cc2c(cc1Cl)NCNS2(=O)=O</smiles>

(I)

In (I), the six-membered ring $\mathrm{N} 1-\mathrm{S} 1-\mathrm{C} 3-\mathrm{C} 2-\mathrm{N} 2-\mathrm{C} 1$ in HCT displays a puckered conformation, atom $\mathrm{N} 1$ having a

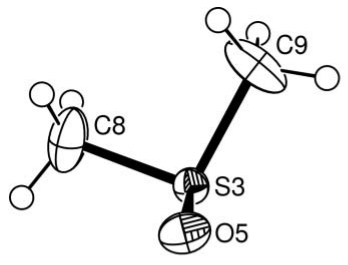

Received 23 March 2006 Accepted 6 May 2006

\section{Figure 1}

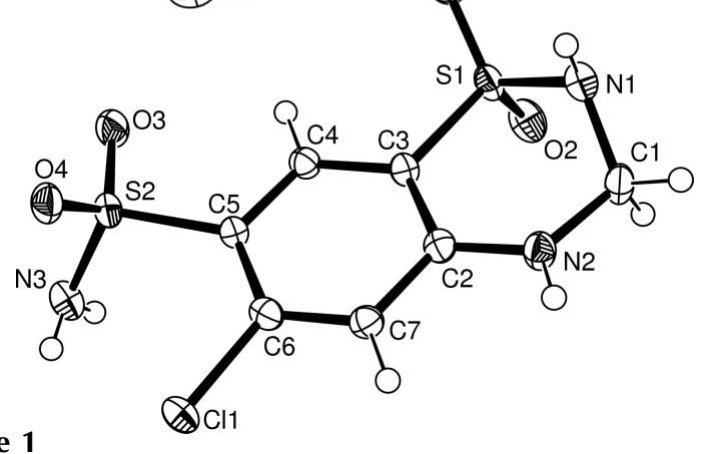

Plot of the asymmetric-unit contents with the atom-numbering scheme. Displacement ellipsoids are drawn at the $50 \%$ probability level. 


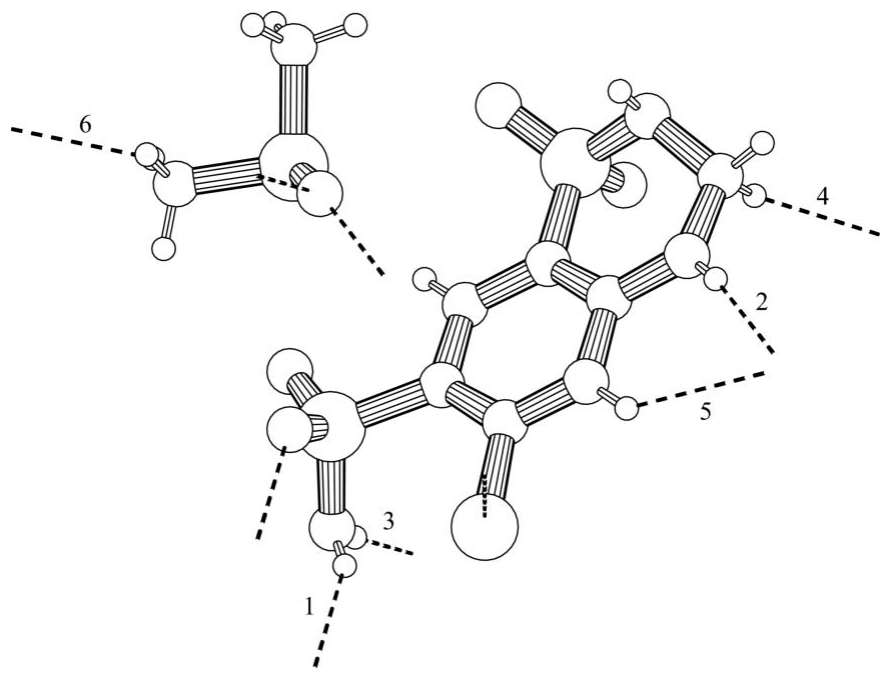

Figure 2

Intermolecular interactions in (I). Dashed lines indicate hydrogen bonds and unique contacts are labelled as follows: $1=\mathrm{N} 3 \cdots \mathrm{O} 4,3.004(3) \AA$, O4 in the molecule at $(-x, 2-y, 2-z) ; 2=\mathrm{N} 2 \cdots \mathrm{O} 5,2.806(3) \AA$, O5 in the molecule at $(1-x, 1-y, 2-z) ; 3=\mathrm{N} 3 \cdots \mathrm{O} 5,2.776(3) \AA$; O5 in the molecule at $(-1+x, y, z) ; 4=\mathrm{C} 1 \cdots \mathrm{O} 4,3.347$ (3) $\mathrm{A}, \mathrm{O} 4$ in the molecule at $(x,-1+y, z) ; 5=\mathrm{C} 7 \cdots \mathrm{O} 5,3.289(3) \AA$, O5 in the molecule at $(1-x$, $1-y, 2-z) ; 6=\mathrm{C} 8 \cdots \mathrm{O} 3,3.228(4) \mathrm{A}, \mathrm{O} 3$ in the molecule at $(1-x, 2-y$, $1-z$ ). Contacts calculated and illustrated using PLATON (Spek, 2003; program version 150306)

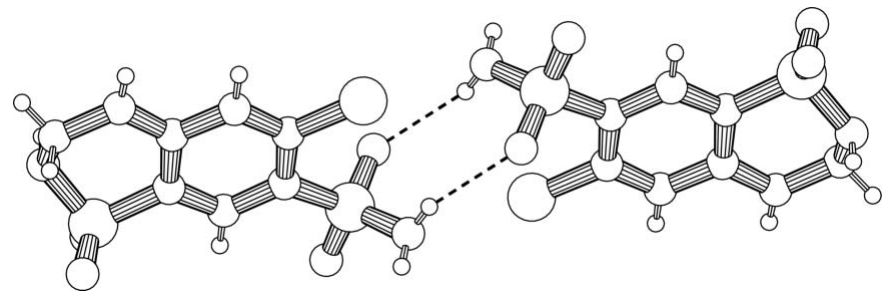

Figure 3

The $R_{2}^{2}(8)$ hydrogen-bonded motif in the crystal structure of (I).

deviation of 0.622 (2) $\AA$ from the least-squares plane through atoms $\mathrm{C} 2-\mathrm{C} 7$. The sulfonamide side chain adopts an $\mathrm{N} 3-\mathrm{S} 2-$ $\mathrm{C} 5-\mathrm{C} 6$ torsion angle of $-62.0(2)^{\circ}$, such that $\mathrm{O} 3$ eclipses $\mathrm{H} 4$, and atoms $\mathrm{O} 4$ and $\mathrm{N} 3$ are staggered with respect to $\mathrm{Cl} 1$.

The crystal structure is stabilized by three $\mathrm{N}-\mathrm{H} \cdots \mathrm{O}$ hydrogen bonds interconnecting (a) HCT molecules (Fig. 2, contact 1) and forming an $R_{2}^{2}(8)$ (Etter, 1990) centrosymmetric dimer (Fig. 3), and (b) HCT and two DMSO molecules (Fig. 2, contacts 2 and 3 ).

The aromatic ring formed by atoms $\mathrm{C} 2-\mathrm{C} 7$ is involved in two offset face-to-face $\pi-\pi$ interactions between nearestneighbour HCT molecules with centroid-centroid distances/ perpendicular distances between the corresponding planes equal to $4.354(2) / 3.58 \AA$ (centroid at $-x, 1-y, 2-z$ ) and 4.466 (2)/3.57 $\AA$ (centroid at $1-x, 1-y, 2-z$ ). The HCT aromatic rings form a stacked arrangement in the direction of the $a$ axis. The structure also contains three $\mathrm{C}-\mathrm{H} \cdots \mathrm{O}$ contacts between HCT and HCT (Fig.2, contact 4) and between HCT and DMSO (contacts 5 and 6).

\section{Experimental}

A single-crystal sample of the title compound was recrystallized by slow evaporation of a dimethyl sulfoxide solution at $278 \mathrm{~K}$.

Crystal data

$\mathrm{C}_{7} \mathrm{H}_{8} \mathrm{ClN}_{3} \mathrm{O}_{4} \mathrm{~S}_{2} \cdot \mathrm{C}_{2} \mathrm{H}_{6} \mathrm{OS}$

$M_{r}=375.86$

Triclinic, $P \overline{1}$

$a=7.5068(4) \AA$

$b=9.8272(5) \AA$

$c=10.7311(6) \mathrm{A}$

$\alpha=85.639(3)^{\circ}$

$\beta=73.896(3)^{\circ}$

$\gamma=80.246(3)^{\circ}$

$$
\begin{aligned}
& V=749.23(7) \AA^{3} \\
& Z=2 \\
& D_{x}=1.666 \mathrm{Mg} \mathrm{m}^{-3} \\
& \text { Mo } K \alpha \text { radiation } \\
& \mu=0.70 \mathrm{~mm}^{-1} \\
& T=123(2) \mathrm{K} \\
& \text { Cut fragment, colourless } \\
& 0.28 \times 0.28 \times 0.10 \mathrm{~mm}
\end{aligned}
$$

\section{Data collection}

Nonius KappaCCD diffractometer $\varphi$ and $\omega$ scans

Absorption correction: none

10594 measured reflections

$$
\begin{aligned}
& 3267 \text { independent reflections } \\
& 2669 \text { reflections with } I>2 \sigma(I) \\
& R_{\text {int }}=0.055 \\
& \theta_{\max }=27.2^{\circ}
\end{aligned}
$$

\section{Refinement}

Refinement on $F^{2}$

$R\left[F^{2}>2 \sigma\left(F^{2}\right)\right]=0.040$

$w R\left(F^{2}\right)=0.110$

$S=1.03$

3267 reflections

208 parameters

$\mathrm{H}$ atoms treated by a mixture of independent and constrained refinement
Table 1

Hydrogen-bond geometry $\left(\AA{ }^{\circ}\right)$.

\begin{tabular}{lllll}
\hline$D-\mathrm{H} \cdots A$ & $D-\mathrm{H}$ & $\mathrm{H} \cdots A$ & $D \cdots A$ & $D-\mathrm{H} \cdots A$ \\
\hline $\mathrm{N} 3-\mathrm{H} 4 N \cdots \mathrm{O} 4^{\mathrm{i}}$ & $0.80(3)$ & $2.27(3)$ & $3.004(3)$ & $153(3)$ \\
$\mathrm{N} 2-\mathrm{H} 2 N \cdots 5^{\text {ii }}$ & $0.81(3)$ & $2.02(3)$ & $2.806(3)$ & $164(3)$ \\
$\mathrm{N} 3-\mathrm{H} 3 N \cdots 5^{\mathrm{iii}}$ & $0.83(3)$ & $1.95(3)$ & $2.776(3)$ & $172(3)$ \\
$\mathrm{C} 1-\mathrm{H} 1 A \cdots 4^{\text {iv }}$ & 0.99 & 2.46 & $3.347(3)$ & 149 \\
$\mathrm{C} 7-\mathrm{H} 7 \cdots$ O $^{\text {ii }}$ & 0.95 & 2.56 & $3.289(3)$ & 134 \\
$\mathrm{C} 8-\mathrm{H} 8 B \cdots 3^{\mathrm{v}}$ & 0.98 & 2.53 & $3.228(4)$ & 128
\end{tabular}

Symmetry codes: (i) $-x,-y+2,-z+2$; (ii) $-x+1,-y+1,-z+2$; (iii) $x-1, y, z$; (iv) $x, y-1, z ;$ (v) $-x+1,-y+2,-z+1$.

$\mathrm{H}$ atoms bonded to $\mathrm{N}$ atoms were found in difference maps and refined isotropically, but all other $\mathrm{H}$ atoms were constrained to idealized geometry using a riding model; $U_{\text {iso }}(\mathrm{H})=1.2 U_{\text {eq }}(\mathrm{C})$ and $\mathrm{C}-$ $\mathrm{H}=0.95(\mathrm{CH}$ group $)$ or $0.99 \AA\left(\mathrm{CH}_{2}\right.$ groups $)$.

Data collection: COLLECT (Hooft, 1988) and DENZO (Otwinowski \& Minor, 1997); cell refinement: DENZO and COLLECT; data reduction: $D E N Z O$; $\operatorname{program}(\mathrm{s})$ used to solve structure: SHELXS97 (Sheldrick, 1997); program(s) used to refine structure: SHELXL97 (Sheldrick, 1997); molecular graphics: PLATON (Spek, 2003); software used to prepare material for publication: SHELXL97.

We thank the Basic Technology programme of the UK Research Councils for funding this work under the project 


\section{organic papers}

Control and Prediction of the Organic Solid State (URL: www.cposs.org.uk).

\section{References}

Dupont, L. \& Dideberg, O. (1972). Acta Cryst. B28, 2340-2347.

Etter, M. C. (1990). Acc. Chem. Res. 23, 120-126.

Florence, A. J., Baumgartner, B., Weston, C., Shankland, N., Kennedy, A. R., Shankland, K. \& David, W. I. F. (2003). J. Pharm. Sci. 92, 1930-1938.
Florence, A. J., Johnston, A., Fernandes, P., Shankland, K., Stevens, H. N. E., Osmundsen, S. \& Mullen, A. B. (2005). Acta Cryst. E61, o2798o2800.

Hooft, R. (1988). COLLECT. Nonius BV, Delft, The Netherlands.

Otwinowski, Z. \& Minor, W. (1997). Methods in Enzymology, Vol. 276, Macromolecular Crystallography, Part A, edited by C. W. Carter Jr \& R. M. Sweet, pp 307-326. New York: Academic Press.

Sheldrick, G. M. (1997). SHELXS97 and SHELXL97. University of Göttingen, Germany.

Spek, A. L. (2003). J. Appl. Cryst. 36, 7-13. 


\title{
supporting information
}

Acta Cryst. (2006). E62, o2288-o2290 [https://doi.org/10.1107/S1600536806016734]

\section{Hydrochlorothiazide dimethyl sulfoxide solvate}

\author{
Andrea Johnston, Alastair J. Florence and Alan R. Kennedy
}

Hydrochlorothiazide dimethyl sulfoxide solvate

Crystal data

$\mathrm{C}_{7} \mathrm{H}_{8} \mathrm{ClN}_{3} \mathrm{O}_{4} \mathrm{~S}_{2} \cdot \mathrm{C}_{2} \mathrm{H}_{6} \mathrm{OS}$

$M_{r}=375.86$

Triclinic, $P \overline{1}$

Hall symbol: -P 1

$a=7.5068(4) \AA$

$b=9.8272(5) \AA$

$c=10.7311(6) \AA$

$\alpha=85.639(3)^{\circ}$

$\beta=73.896(3)^{\circ}$

$\gamma=80.246(3)^{\circ}$

$V=749.23(7) \AA^{3}$

Data collection

Nonius KappaCCD

diffractometer

Radiation source: fine-focus sealed tube

Graphite monochromator

$\varphi$ and $\omega$ scans

10594 measured reflections

3267 independent reflections

\section{Refinement}

Refinement on $F^{2}$

Least-squares matrix: full

$R\left[F^{2}>2 \sigma\left(F^{2}\right)\right]=0.040$

$w R\left(F^{2}\right)=0.110$

$S=1.04$

3267 reflections

208 parameters

0 restraints

Primary atom site location: structure-invariant direct methods
$Z=2$

$F(000)=388$

$D_{\mathrm{x}}=1.666 \mathrm{Mg} \mathrm{m}^{-3}$

Mo $K \alpha$ radiation, $\lambda=0.71073 \AA$

Cell parameters from 2971 reflections

$\theta=2.9-27.1^{\circ}$

$\mu=0.70 \mathrm{~mm}^{-1}$

$T=123 \mathrm{~K}$

Cut fragment, colourless

$0.28 \times 0.28 \times 0.10 \mathrm{~mm}$

2669 reflections with $I>2 \sigma(I)$

$R_{\text {int }}=0.055$

$\theta_{\max }=27.2^{\circ}, \theta_{\min }=2.9^{\circ}$

$h=-9 \rightarrow 9$

$k=-11 \rightarrow 12$

$l=-13 \rightarrow 13$
Secondary atom site location: difference Fourier map

Hydrogen site location: inferred from neighbouring sites

$\mathrm{H}$ atoms treated by a mixture of independent and constrained refinement

$w=1 /\left[\sigma^{2}\left(F_{0}^{2}\right)+(0.0592 P)^{2}+0.534 P\right]$ where $P=\left(F_{\mathrm{o}}{ }^{2}+2 F_{\mathrm{c}}{ }^{2}\right) / 3$

$(\Delta / \sigma)_{\max }=0.001$

$\Delta \rho_{\max }=0.47$ e $\AA^{-3}$

$\Delta \rho_{\min }=-0.48$ e $\AA^{-3}$

Special details

Experimental. Although no formal absorption correction was applied, the standard Kappa CCD methodology involves collecting a large number of redundant reflections and processing via SCALEPACK. This effectively introduces a multiscan type correction suitable for weakly absorbing molecules. 
Geometry. All e.s.d.'s (except the e.s.d. in the dihedral angle between two 1.s. planes) are estimated using the full covariance matrix. The cell e.s.d.'s are taken into account individually in the estimation of e.s.d.'s in distances, angles and torsion angles; correlations between e.s.d.'s in cell parameters are only used when they are defined by crystal symmetry. An approximate (isotropic) treatment of cell e.s.d.'s is used for estimating e.s.d.'s involving 1.s. planes.

Refinement. Refinement of $F^{2}$ against ALL reflections. The weighted $R$-factor $w R$ and goodness of fit $S$ are based on $F^{2}$, conventional $R$-factors $R$ are based on $F$, with $F$ set to zero for negative $F^{2}$. The threshold expression of $F^{2}>\sigma\left(F^{2}\right)$ is used only for calculating $R$-factors(gt) $e t c$. and is not relevant to the choice of reflections for refinement. $R$-factors based on $F^{2}$ are statistically about twice as large as those based on $F$, and $R$ - factors based on ALL data will be even larger.

Fractional atomic coordinates and isotropic or equivalent isotropic displacement parameters $\left(\AA^{2}\right)$

\begin{tabular}{|c|c|c|c|c|}
\hline & $x$ & $y$ & $z$ & $U_{\text {iso }} * / U_{\text {eq }}$ \\
\hline $\mathrm{Cl1}$ & $0.02575(8)$ & $0.73342(6)$ & $1.11711(6)$ & $0.02319(16)$ \\
\hline S1 & $0.51163(9)$ & $0.34312(6)$ & $0.67153(6)$ & $0.02145(16)$ \\
\hline $\mathrm{S} 2$ & $0.15774(9)$ & $0.85850(6)$ & $0.81869(6)$ & $0.02141(16)$ \\
\hline $\mathrm{S} 3$ & $0.69526(9)$ & $0.76246(6)$ & $0.63385(6)$ & $0.02243(17)$ \\
\hline $\mathrm{O} 1$ & $0.6400(3)$ & 0.41391 (19) & $0.57579(18)$ & $0.0319(4)$ \\
\hline $\mathrm{O} 2$ & $0.3873(3)$ & 0.27152 (19) & $0.63320(18)$ & $0.0300(4)$ \\
\hline $\mathrm{O} 3$ & $0.2162(3)$ & $0.86589(18)$ & $0.67974(17)$ & $0.0295(4)$ \\
\hline $\mathrm{O} 4$ & $0.2406(3)$ & $0.93652(17)$ & $0.89048(18)$ & $0.0267(4)$ \\
\hline $\mathrm{O} 5$ & $0.7163(3)$ & $0.7637(2)$ & $0.77073(17)$ & $0.0322(4)$ \\
\hline N1 & 0.6358 & $0.2308(2)$ & $0.7473(2)$ & $0.0245(5)$ \\
\hline N2 & 0.4255 & $0.2776(2)$ & $0.9606(2)$ & $0.0225(5)$ \\
\hline N3 & $-0.0632(3)$ & $0.9093(2)$ & $0.8633(2)$ & $0.0248(5)$ \\
\hline $\mathrm{C} 1$ & $0.5231(4)$ & $0.1724(2)$ & $0.8661(2)$ & $0.0236(5)$ \\
\hline $\mathrm{H} 1 \mathrm{~A}$ & 0.4299 & 0.1247 & 0.8446 & $0.028^{*}$ \\
\hline H1B & 0.6056 & 0.1030 & 0.9050 & $0.028 *$ \\
\hline $\mathrm{C} 2$ & 0.3507 & $0.4058(2)$ & $0.9263(2)$ & $0.0182(5)$ \\
\hline $\mathrm{C} 3$ & $0.3807(3)$ & $0.4550(2)$ & $0.7960(2)$ & $0.0181(5)$ \\
\hline $\mathrm{C} 4$ & $0.3152(3)$ & $0.5911(2)$ & $0.7652(2)$ & $0.0190(5)$ \\
\hline $\mathrm{H} 4$ & 0.3415 & 0.6224 & 0.6770 & $0.023 *$ \\
\hline $\mathrm{C} 5$ & $0.2114(3)$ & $0.6819(2)$ & $0.8622(2)$ & $0.0179(5)$ \\
\hline C6 & $0.1710(3)$ & $0.6301(2)$ & $0.9914(2)$ & $0.0192(5)$ \\
\hline $\mathrm{C} 7$ & 0.2385 & $0.4977(2)$ & $1.0242(2)$ & $0.0193(5)$ \\
\hline H7 & 0.2103 & 0.4671 & 1.1127 & $0.023 *$ \\
\hline $\mathrm{C} 8$ & $0.6947(6)$ & $0.9341(3)$ & $0.5754(3)$ & $0.0489(9)$ \\
\hline H8A & 0.5787 & 0.9911 & 0.6223 & $0.073 *$ \\
\hline H8B & 0.7023 & 0.9384 & 0.4826 & $0.073 *$ \\
\hline $\mathrm{H} 8 \mathrm{C}$ & 0.8029 & 0.9687 & 0.5888 & $0.073 *$ \\
\hline C9 & $0.9207(5)$ & $0.6894(4)$ & 0.5400 & $0.0517(10)$ \\
\hline H9A & 1.0142 & 0.7440 & 0.5485 & $0.078^{*}$ \\
\hline H9B & 0.9206 & 0.6894 & 0.4487 & $0.078^{*}$ \\
\hline $\mathrm{H} 9 \mathrm{C}$ & 0.9518 & 0.5942 & 0.5710 & $0.078 *$ \\
\hline $\mathrm{H} 1 \mathrm{~N}$ & $0.729(5)$ & $0.264(3)$ & $0.758(3)$ & $0.036(9)^{*}$ \\
\hline $\mathrm{H} 2 \mathrm{~N}$ & 0.407 (4) & $0.261(3)$ & $1.038(3)$ & $0.023(7)^{*}$ \\
\hline $\mathrm{H} 3 \mathrm{~N}$ & $-0.131(4)$ & $0.872(3)$ & $0.831(3)$ & $0.023(8)^{*}$ \\
\hline $\mathrm{H} 4 \mathrm{~N}$ & $-0.107(4)$ & $0.923(3)$ & $0.939(3)$ & $0.026(8)^{*}$ \\
\hline
\end{tabular}


Atomic displacement parameters $\left(\AA^{2}\right)$

\begin{tabular}{lllllll}
\hline & $U^{11}$ & $U^{22}$ & $U^{33}$ & $U^{12}$ & $U^{13}$ & $U^{23}$ \\
\hline C11 & $0.0229(3)$ & $0.0246(3)$ & $0.0208(3)$ & $0.0002(2)$ & $-0.0042(2)$ & $-0.0075(2)$ \\
S1 & $0.0253(3)$ & $0.0181(3)$ & $0.0191(3)$ & $0.0034(2)$ & $-0.0060(2)$ & $-0.0041(2)$ \\
S2 & $0.0236(3)$ & $0.0151(3)$ & $0.0238(3)$ & $0.0000(2)$ & $-0.0048(2)$ & $-0.0020(2)$ \\
S3 & $0.0241(3)$ & $0.0226(3)$ & $0.0207(3)$ & $-0.0030(2)$ & $-0.0065(2)$ & $-0.0015(2)$ \\
O1 & $0.0332(11)$ & $0.0284(10)$ & $0.0257(10)$ & $0.0020(8)$ & $0.0022(8)$ & $-0.0011(8)$ \\
O2 & $0.0321(10)$ & $0.0292(10)$ & $0.0316(10)$ & $-0.0003(8)$ & $-0.0132(8)$ & $-0.0109(8)$ \\
O3 & $0.0374(11)$ & $0.0212(9)$ & $0.0238(10)$ & $0.0024(8)$ & $-0.0035(8)$ & $0.0026(7)$ \\
O4 & $0.0271(10)$ & $0.0185(8)$ & $0.0346(10)$ & $-0.0042(7)$ & $-0.0066(8)$ & $-0.0057(7)$ \\
O5 & $0.0353(11)$ & $0.0433(11)$ & $0.0196(9)$ & $-0.0103(9)$ & $-0.0088(8)$ & $0.0038(8)$ \\
N1 & $0.0244(11)$ & $0.0207(10)$ & $0.0276(12)$ & $0.0008(9)$ & $-0.0076(9)$ & $-0.0026(9)$ \\
N2 & $0.0292(12)$ & $0.0183(10)$ & $0.0194(11)$ & $-0.0009(8)$ & $-0.0075(9)$ & $-0.0001(8)$ \\
N3 & $0.0245(12)$ & $0.0245(11)$ & $0.0262(13)$ & $0.0015(9)$ & $-0.0100(10)$ & $-0.0064(9)$ \\
C1 & $0.0305(14)$ & $0.0148(11)$ & $0.0252(13)$ & $-0.0007(10)$ & $-0.0089(11)$ & $0.0001(9)$ \\
C2 & $0.0172(11)$ & $0.0178(11)$ & $0.0214(12)$ & $-0.0040(9)$ & $-0.0068(9)$ & $-0.0022(9)$ \\
C3 & $0.0195(11)$ & $0.0159(11)$ & $0.0189(12)$ & $-0.0012(9)$ & $-0.0058(9)$ & $-0.0024(9)$ \\
C4 & $0.0196(12)$ & $0.0189(11)$ & $0.0177(11)$ & $-0.0017(9)$ & $-0.0045(9)$ & $-0.0012(9)$ \\
C5 & $0.0184(11)$ & $0.0156(11)$ & $0.0189(11)$ & $-0.0015(9)$ & $-0.0042(9)$ & $-0.0019(9)$ \\
C6 & $0.0168(11)$ & $0.0211(11)$ & $0.0209(12)$ & $-0.0047(9)$ & $-0.0043(9)$ & $-0.0064(9)$ \\
C7 & $0.0208(12)$ & $0.0203(11)$ & $0.0173(11)$ & $-0.0047(9)$ & $-0.0048(9)$ & $-0.0014(9)$ \\
C8 & $0.086(3)$ & $0.0267(15)$ & $0.0400(18)$ & $-0.0107(16)$ & $-0.0281(18)$ & $0.0061(13)$ \\
C9 & $0.0374(18)$ & $0.072(2)$ & $0.0395(19)$ & $0.0171(17)$ & $-0.0091(15)$ & $-0.0262(17)$ \\
& & & & & & \\
\hline
\end{tabular}

Geometric parameters $\left(\AA,{ }^{\circ}\right)$

\begin{tabular}{llll}
\hline $\mathrm{C} 11-\mathrm{C} 6$ & $1.749(2)$ & $\mathrm{N} 3-\mathrm{H} 4 \mathrm{~N}$ & $0.80(3)$ \\
$\mathrm{S} 1-\mathrm{O} 2$ & $1.4195(19)$ & $\mathrm{C} 1-\mathrm{H} 1 \mathrm{~A}$ & 0.9900 \\
$\mathrm{~S} 1-\mathrm{O} 1$ & $1.427(2)$ & $\mathrm{C} 1-\mathrm{H} 1 \mathrm{~B}$ & 0.9900 \\
$\mathrm{~S} 1-\mathrm{N} 1$ & $1.638(2)$ & $\mathrm{C} 2-\mathrm{C} 3$ & $1.413(3)$ \\
$\mathrm{S} 1-\mathrm{C} 3$ & $1.758(2)$ & $\mathrm{C} 2-\mathrm{C} 7$ & $1.422(3)$ \\
$\mathrm{S} 2-\mathrm{O} 3$ & $1.4327(19)$ & $\mathrm{C} 3-\mathrm{C} 4$ & $1.393(3)$ \\
$\mathrm{S} 2-\mathrm{O} 4$ & $1.4443(19)$ & $\mathrm{C} 4-\mathrm{C} 5$ & $1.394(3)$ \\
$\mathrm{S} 2-\mathrm{N} 3$ & $1.595(2)$ & $\mathrm{C}-\mathrm{H} 4$ & 0.9500 \\
$\mathrm{~S} 2-\mathrm{C} 5$ & $1.771(2)$ & $\mathrm{C} 5-\mathrm{C} 6$ & $1.409(3)$ \\
$\mathrm{S} 3-\mathrm{O} 5$ & $1.5213(18)$ & $\mathrm{C} 6-\mathrm{C} 7$ & $1.371(3)$ \\
$\mathrm{S} 3-\mathrm{C} 8$ & $1.754(3)$ & $\mathrm{C} 7-\mathrm{H} 7$ & 0.9500 \\
$\mathrm{~S} 3-\mathrm{C} 9$ & $1.774(3)$ & $\mathrm{C} 8-\mathrm{H} 8 \mathrm{~A}$ & 0.9800 \\
$\mathrm{~N} 1-\mathrm{C} 1$ & $1.458(3)$ & $\mathrm{C} 8-\mathrm{H} 8 \mathrm{~B}$ & 0.9800 \\
$\mathrm{~N} 1-\mathrm{H} 1 \mathrm{~N}$ & $0.85(3)$ & $\mathrm{C} 8-\mathrm{H} 8 \mathrm{C}$ & 0.9800 \\
$\mathrm{~N} 2-\mathrm{C} 2$ & $1.358(3)$ & $\mathrm{C} 9-\mathrm{H} 9 \mathrm{~A}$ & 0.9800 \\
$\mathrm{~N} 2-\mathrm{C} 1$ & $1.457(3)$ & $\mathrm{C} 9-\mathrm{H} 9 \mathrm{~B}$ & 0.9800 \\
$\mathrm{~N} 2-\mathrm{H} 2 \mathrm{~N}$ & $0.81(3)$ & $\mathrm{C} 9-\mathrm{H} 9 \mathrm{C}$ & \\
$\mathrm{N} 3-\mathrm{H} 3 \mathrm{~N}$ & $0.83(3)$ & & $123.0(2)$ \\
& & & $119.7(2)$ \\
$\mathrm{O} 2-\mathrm{S} 1-\mathrm{O} 1$ & $119.64(12)$ & $\mathrm{N} 2-\mathrm{C} 2-\mathrm{C} 3$ & \\
$\mathrm{O} 2-\mathrm{S} 1-\mathrm{N} 1$ & $107.28(12)$ & $\mathrm{N} 2-\mathrm{C} 2-\mathrm{C} 7$ &
\end{tabular}




\begin{tabular}{|c|c|c|c|}
\hline $\mathrm{O} 1-\mathrm{S} 1-\mathrm{N} 1$ & $107.26(12)$ & $\mathrm{C} 3-\mathrm{C} 2-\mathrm{C} 7$ & $117.3(2)$ \\
\hline $\mathrm{O} 2-\mathrm{S} 1-\mathrm{C} 3$ & $108.77(11)$ & $\mathrm{C} 4-\mathrm{C} 3-\mathrm{C} 2$ & $121.4(2)$ \\
\hline $\mathrm{O} 1-\mathrm{S} 1-\mathrm{C} 3$ & $110.26(11)$ & $\mathrm{C} 4-\mathrm{C} 3-\mathrm{S} 1$ & $119.71(18)$ \\
\hline $\mathrm{N} 1-\mathrm{S} 1-\mathrm{C} 3$ & $102.16(11)$ & $\mathrm{C} 2-\mathrm{C} 3-\mathrm{S} 1$ & $118.87(17)$ \\
\hline $\mathrm{O} 3-\mathrm{S} 2-\mathrm{O} 4$ & $118.94(12)$ & $\mathrm{C} 3-\mathrm{C} 4-\mathrm{C} 5$ & $120.8(2)$ \\
\hline $\mathrm{O} 3-\mathrm{S} 2-\mathrm{N} 3$ & $107.80(13)$ & $\mathrm{C} 3-\mathrm{C} 4-\mathrm{H} 4$ & 119.6 \\
\hline $\mathrm{O} 4-\mathrm{S} 2-\mathrm{N} 3$ & $106.60(12)$ & $\mathrm{C} 5-\mathrm{C} 4-\mathrm{H} 4$ & 119.6 \\
\hline $\mathrm{O} 3-\mathrm{S} 2-\mathrm{C} 5$ & $105.53(11)$ & $\mathrm{C} 4-\mathrm{C} 5-\mathrm{C} 6$ & $117.7(2)$ \\
\hline $\mathrm{O} 4-\mathrm{S} 2-\mathrm{C} 5$ & $107.25(11)$ & $\mathrm{C} 4-\mathrm{C} 5-\mathrm{S} 2$ & $118.49(18)$ \\
\hline $\mathrm{N} 3-\mathrm{S} 2-\mathrm{C} 5$ & $110.66(12)$ & $\mathrm{C} 6-\mathrm{C} 5-\mathrm{S} 2$ & $123.52(17)$ \\
\hline $\mathrm{O} 5-\mathrm{S} 3-\mathrm{C} 8$ & $105.16(14)$ & $\mathrm{C} 7-\mathrm{C} 6-\mathrm{C} 5$ & $122.4(2)$ \\
\hline $\mathrm{O} 5-\mathrm{S} 3-\mathrm{C} 9$ & $104.42(14)$ & $\mathrm{C} 7-\mathrm{C} 6-\mathrm{Cl} 1$ & 117.17 (19) \\
\hline $\mathrm{C} 8-\mathrm{S} 3-\mathrm{C} 9$ & $99.1(2)$ & $\mathrm{C} 5-\mathrm{C} 6-\mathrm{Cl1}$ & $120.43(18)$ \\
\hline $\mathrm{C} 1-\mathrm{N} 1-\mathrm{S} 1$ & $113.31(18)$ & $\mathrm{C} 6-\mathrm{C} 7-\mathrm{C} 2$ & $120.2(2)$ \\
\hline $\mathrm{C} 1-\mathrm{N} 1-\mathrm{H} 1 \mathrm{~N}$ & $113(2)$ & $\mathrm{C} 6-\mathrm{C} 7-\mathrm{H} 7$ & 119.9 \\
\hline $\mathrm{S} 1-\mathrm{N} 1-\mathrm{H} 1 \mathrm{~N}$ & $111(2)$ & $\mathrm{C} 2-\mathrm{C} 7-\mathrm{H} 7$ & 119.9 \\
\hline $\mathrm{C} 2-\mathrm{N} 2-\mathrm{C} 1$ & $122.8(2)$ & $\mathrm{S} 3-\mathrm{C} 8-\mathrm{H} 8 \mathrm{~A}$ & 109.5 \\
\hline $\mathrm{C} 2-\mathrm{N} 2-\mathrm{H} 2 \mathrm{~N}$ & $116(2)$ & $\mathrm{S} 3-\mathrm{C} 8-\mathrm{H} 8 \mathrm{~B}$ & 109.5 \\
\hline $\mathrm{C} 1-\mathrm{N} 2-\mathrm{H} 2 \mathrm{~N}$ & $121(2)$ & $\mathrm{H} 8 \mathrm{~A}-\mathrm{C} 8-\mathrm{H} 8 \mathrm{~B}$ & 109.5 \\
\hline $\mathrm{S} 2-\mathrm{N} 3-\mathrm{H} 3 \mathrm{~N}$ & $118(2)$ & $\mathrm{S} 3-\mathrm{C} 8-\mathrm{H} 8 \mathrm{C}$ & 109.5 \\
\hline $\mathrm{S} 2-\mathrm{N} 3-\mathrm{H} 4 \mathrm{~N}$ & $116(2)$ & $\mathrm{H} 8 \mathrm{~A}-\mathrm{C} 8-\mathrm{H} 8 \mathrm{C}$ & 109.5 \\
\hline $\mathrm{H} 3 \mathrm{~N}-\mathrm{N} 3-\mathrm{H} 4 \mathrm{~N}$ & $113(3)$ & $\mathrm{H} 8 \mathrm{~B}-\mathrm{C} 8-\mathrm{H} 8 \mathrm{C}$ & 109.5 \\
\hline $\mathrm{N} 2-\mathrm{C} 1-\mathrm{N} 1$ & $112.2(2)$ & $\mathrm{S} 3-\mathrm{C} 9-\mathrm{H} 9 \mathrm{~A}$ & 109.5 \\
\hline $\mathrm{N} 2-\mathrm{C} 1-\mathrm{H} 1 \mathrm{~A}$ & 109.2 & $\mathrm{~S} 3-\mathrm{C} 9-\mathrm{H} 9 \mathrm{~B}$ & 109.5 \\
\hline $\mathrm{N} 1-\mathrm{C} 1-\mathrm{H} 1 \mathrm{~A}$ & 109.2 & $\mathrm{H} 9 \mathrm{~A}-\mathrm{C} 9-\mathrm{H} 9 \mathrm{~B}$ & 109.5 \\
\hline $\mathrm{N} 2-\mathrm{C} 1-\mathrm{H} 1 \mathrm{~B}$ & 109.2 & $\mathrm{~S} 3-\mathrm{C} 9-\mathrm{H} 9 \mathrm{C}$ & 109.5 \\
\hline $\mathrm{N} 1-\mathrm{C} 1-\mathrm{H} 1 \mathrm{~B}$ & 109.2 & $\mathrm{H} 9 \mathrm{~A}-\mathrm{C} 9-\mathrm{H} 9 \mathrm{C}$ & 109.5 \\
\hline $\mathrm{H} 1 \mathrm{~A}-\mathrm{C} 1-\mathrm{H} 1 \mathrm{~B}$ & 107.9 & $\mathrm{H} 9 \mathrm{~B}-\mathrm{C} 9-\mathrm{H} 9 \mathrm{C}$ & 109.5 \\
\hline $\mathrm{O} 2-\mathrm{S} 1-\mathrm{N} 1-\mathrm{C} 1$ & $-63.1(2)$ & $\mathrm{S} 1-\mathrm{C} 3-\mathrm{C} 4-\mathrm{C} 5$ & $-179.87(18)$ \\
\hline $\mathrm{O} 1-\mathrm{S} 1-\mathrm{N} 1-\mathrm{C} 1$ & $167.15(17)$ & $\mathrm{C} 3-\mathrm{C} 4-\mathrm{C} 5-\mathrm{C} 6$ & $-1.8(4)$ \\
\hline $\mathrm{C} 3-\mathrm{S} 1-\mathrm{N} 1-\mathrm{C} 1$ & $51.2(2)$ & $\mathrm{C} 3-\mathrm{C} 4-\mathrm{C} 5-\mathrm{S} 2$ & $172.14(19)$ \\
\hline $\mathrm{C} 2-\mathrm{N} 2-\mathrm{C} 1-\mathrm{N} 1$ & $37.5(3)$ & $\mathrm{O} 3-\mathrm{S} 2-\mathrm{C} 5-\mathrm{C} 4$ & $8.1(2)$ \\
\hline $\mathrm{S} 1-\mathrm{N} 1-\mathrm{C} 1-\mathrm{N} 2$ & $-61.1(3)$ & $\mathrm{O} 4-\mathrm{S} 2-\mathrm{C} 5-\mathrm{C} 4$ & $-119.65(19)$ \\
\hline $\mathrm{C} 1-\mathrm{N} 2-\mathrm{C} 2-\mathrm{C} 3$ & $-8.2(4)$ & $\mathrm{N} 3-\mathrm{S} 2-\mathrm{C} 5-\mathrm{C} 4$ & $124.5(2)$ \\
\hline $\mathrm{C} 1-\mathrm{N} 2-\mathrm{C} 2-\mathrm{C} 7$ & $172.6(2)$ & $\mathrm{O} 3-\mathrm{S} 2-\mathrm{C} 5-\mathrm{C} 6$ & $-178.3(2)$ \\
\hline $\mathrm{N} 2-\mathrm{C} 2-\mathrm{C} 3-\mathrm{C} 4$ & $-174.4(2)$ & $\mathrm{O} 4-\mathrm{S} 2-\mathrm{C} 5-\mathrm{C} 6$ & $53.9(2)$ \\
\hline $\mathrm{C} 7-\mathrm{C} 2-\mathrm{C} 3-\mathrm{C} 4$ & $4.7(3)$ & $\mathrm{N} 3-\mathrm{S} 2-\mathrm{C} 5-\mathrm{C} 6$ & $-62.0(2)$ \\
\hline $\mathrm{N} 2-\mathrm{C} 2-\mathrm{C} 3-\mathrm{S} 1$ & $3.1(3)$ & $\mathrm{C} 4-\mathrm{C} 5-\mathrm{C} 6-\mathrm{C} 7$ & $3.7(4)$ \\
\hline $\mathrm{C} 7-\mathrm{C} 2-\mathrm{C} 3-\mathrm{S} 1$ & $-177.79(17)$ & $\mathrm{S} 2-\mathrm{C} 5-\mathrm{C} 6-\mathrm{C} 7$ & $-169.91(19)$ \\
\hline $\mathrm{O} 2-\mathrm{S} 1-\mathrm{C} 3-\mathrm{C} 4$ & $-92.3(2)$ & $\mathrm{C} 4-\mathrm{C} 5-\mathrm{C} 6-\mathrm{Cl1}$ & $-174.82(18)$ \\
\hline $\mathrm{O} 1-\mathrm{S} 1-\mathrm{C} 3-\mathrm{C} 4$ & $40.7(2)$ & $\mathrm{S} 2-\mathrm{C} 5-\mathrm{C} 6-\mathrm{Cl1}$ & $11.6(3)$ \\
\hline $\mathrm{N} 1-\mathrm{S} 1-\mathrm{C} 3-\mathrm{C} 4$ & $154.5(2)$ & $\mathrm{C} 5-\mathrm{C} 6-\mathrm{C} 7-\mathrm{C} 2$ & $-1.3(4)$ \\
\hline $\mathrm{O} 2-\mathrm{S} 1-\mathrm{C} 3-\mathrm{C} 2$ & $90.2(2)$ & $\mathrm{C} 11-\mathrm{C} 6-\mathrm{C} 7-\mathrm{C} 2$ & $177.25(18)$ \\
\hline $\mathrm{O} 1-\mathrm{S} 1-\mathrm{C} 3-\mathrm{C} 2$ & $-136.8(2)$ & $\mathrm{N} 2-\mathrm{C} 2-\mathrm{C} 7-\mathrm{C} 6$ & $176.3(2)$ \\
\hline $\mathrm{N} 1-\mathrm{S} 1-\mathrm{C} 3-\mathrm{C} 2$ & $-23.0(2)$ & $\mathrm{C} 3-\mathrm{C} 2-\mathrm{C} 7-\mathrm{C} 6$ & $-2.9(3)$ \\
\hline $\mathrm{C} 2-\mathrm{C} 3-\mathrm{C} 4-\mathrm{C} 5$ & $-2.4(4)$ & & \\
\hline
\end{tabular}


supporting information

Hydrogen-bond geometry $\left(\AA,{ }^{\circ}\right)$

\begin{tabular}{lllll}
\hline$D-\mathrm{H} \cdots A$ & $D-\mathrm{H}$ & $\mathrm{H} \cdots A$ & $D \cdots A$ & $D-\mathrm{H} \cdots A$ \\
\hline $\mathrm{N} 3-\mathrm{H} 4 N \cdots \mathrm{O} 4^{\mathrm{i}}$ & $0.80(3)$ & $2.27(3)$ & $3.004(3)$ & $153(3)$ \\
$\mathrm{N} 2-\mathrm{H} 2 N \cdots 5^{\text {ii }}$ & $0.81(3)$ & $2.02(3)$ & $2.806(3)$ & $164(3)$ \\
$\mathrm{N} 3-\mathrm{H} 3 N \cdots 5^{\text {iii }}$ & $0.83(3)$ & $1.95(3)$ & $2.776(3)$ & $172(3)$ \\
$\mathrm{C} 1-\mathrm{H} 1 A \cdots 4^{\text {iv }}$ & 0.99 & 2.46 & $3.347(3)$ & 149 \\
$\mathrm{C} 7-\mathrm{H} 7 \cdots 5^{\mathrm{ii}}$ & 0.95 & 2.56 & $3.289(3)$ & 134 \\
$\mathrm{C} 8-\mathrm{H} 8 B \cdots 3^{v}$ & 0.98 & 2.53 & $3.228(4)$ & 128 \\
\hline
\end{tabular}

Symmetry codes: (i) $-x,-y+2,-z+2$; (ii) $-x+1,-y+1,-z+2$; (iii) $x-1, y, z$; (iv) $x, y-1, z$; (v) $-x+1,-y+2,-z+1$. 